\title{
Poblamiento, desarrollo, conservación y conflicto en la costa de Jalisco: una revisión histórica
}

\author{
Carlos Gauna Ruíz de León* \\ Universidad de Guadalajara (México)
}

\begin{abstract}
Resumen: La costa de Jalisco tiene una belleza natural impresionante, con escenarios naturales entre playa y tierra que se complementan en un territorio diverso y con enormes potencialidades para el desarrollo de actividades productivas, tanto agropecuarias como de turismo, con la condición de que se sean sostenibles tanto para el medio ambiente como para las comunidades allí asentadas. Desde principios del siglo veinte se han generado proyectos de intervención en la región, algunos fueron abandonados, otros tardaron tanto tiempo que cuando se concluyeron ya no respondían a las nuevas necesidades, y otros provocaron un desarrollo importante en términos sociales y económicos, como lo es Puerto Vallarta. El objetivo del trabajo es hacer un relato histórico, con el mayor número de proyectos que se han gestado en la costa, que permitan comprender su proceso a lo largo del tiempo. Caracterizar a la región a partir de los hechos que allí se gestaron permite entender el estado que guarda, comprender las motivaciones de los actores y la relevancia de sus acciones en la configuración de la realidad actual.
\end{abstract}

Palabras Clave: Desarrollo regional; Turismo; Territorio.

\section{Population, development, conservation and conflict in the Jalisco coast: a historical review}

Abstract: The coast of Jalisco boasts impressive natural beauty, with various different scenarios of combinations of beaches and countryside that offer enormous potential for responsible activities in both tourism and the primary sector, thereby favouring the resident populations and preserving the heritage features. As of the twentieth century onwards, various different projects have been embarked upon most of which were left inconcluded or became redundant, no longer responding to the demands of the times, but some of which, such as Puerto Vallarta, caused significant socio-economic development.. This work is a historical description of the region that highlights these processes in order to fully comprehend the hows, whos and whys of these actions together with their present relevance.

Keywords: Local development; Tourism; Territory.

\section{Introducción}

La costa de Jalisco ha sido un territorio de interés desde hace un siglo, ha sido objeto de diversos procesos de planeación del desarrollo, que configuran lo que hoy es la costa. La determinación del territorio a explicar contempla a los cinco municipios costeros, Puerto Vallarta, Cabo Corrientes, Tomatlán, la Huerta y Cihuatlán, su radio de influencia abarca otros municipios colindantes que se localizan en la sierra o en los estados vecinos de Colima y Nayarit.

La costa de Jalisco tiene una gran riqueza natural, fue el primer motivante para su poblamiento, por ser un territorio con grandes potencialidades de producción agropecuaria y en menor medida pesquera, asentando comunidades que se dedican a estas actividades productivas. Se da a partir de la dotación de tierras a campesinos y de la construcción de infraestructura para permitir la trasferencia de los productos generados hacia los mercados que los demandan.

Profesor de tiempo completo de la Universidad de Guadalajara; Coordinador del posgrado en Ciencias para el Desarrollo, la Sustentabilidad y el Turismo; Miembro del Sistema Nacional de Investigadores.; E-mail: carlosg@cuc.udg.mx 
Por su belleza natural de mar y montaña, se convirtió en una de las principales atracciones para el desarrollo del turismo, un primer impulso se da desde las políticas públicas en la generación de proyectos y construcción y ampliación de infraestructura y posterior por los intereses de los actores privados que generan proyectos de inversión para hacer factibles los emprendimientos productivos de condiciones y tamaños diversos.

Entre ellos Puerto Vallarta uno de los destinos turísticos más emblemáticos, la zona de CareyesCuixmala- Chamela, con resorts de una alta calidad, rodeada de un área natural protegida y que está creciendo con proyectos de características privadas y exclusivas, además de diversos proyectos de baja inversión, que son usados por el turismo nacional de ingresos medios y bajos, el más importante es Melaque-Barra de Navidad.

Los intereses de los actores (ejidatarios, desarrolladores, proteccionistas) generan un proceso dual, el desarrollo de proyectos productivos que configuran el perfil de la actividad económica de la costa y con ello, conflictos por las visiones encontradas, que van desde litigios legales hasta enfrentamientos armados.

Actualmente están en proceso muchos proyectos de intervención en la costa, lo que hace pertinente un análisis de los hechos que han configurado a la región, reconociendo los aciertos y evaluando los fracasos, que sirva para dar certidumbre al futuro deseable.

\section{Metodología}

El trabajo tiene como objetivo reunir la mayor parte de los estudios y aportaciones sobre la costa de Jalisco, que permita tener un conocimiento a partir del recuento de las acciones que se sucedieron en el territorio desde hace más de 100 años. Esto ha permitido integrar un proyecto amplio de investigación que permita explicar la situación de la región y marcar sus potencialidades.

La información se basa en diversas fuentes, las oficiales formuladas por la propia autoridad o encargadas a empresas consultoras, el trabajo académico presentado en libros y revistas de divulgación científica, las publicaciones periódicas en medios impresos y electrónicos y los documentos de carácter mercadológico de las empresas.

En un segundo proceso de búsqueda se trabajo en los buscadores académicos electrónicos que dieran cuenta de información sobre la temática específicas que requerían mejor información y de aportaciones de investigadores que amablemente integraron otros documentos.

Finalmente se consultaron fuentes oficiales sobre indicadores de población y de datos del turismo que permitieron ampliar el contexto de la región.

Una parte importante de este trabajo fue localizar las fuentes teóricas de las cuales se basaron muchas políticas de desarrollo en la región que permiten comprender las referencias al trabajo realizado, pero también en la búsqueda de elementos teóricos actuales que permitan una mejor comprensión del hecho estudiado.

La motivación de construir este relato, surge al no encontrar suficiente información académica documentada de la región, más aun, no existe un relato amplio que integre su historia, tampoco se localizan documentos completos de los proyectos y no existe un banco de información sistemáticamente organizada.

Una condición del trabajo ha sido el carácter holístico de la construcción del conocimiento, la fragmentación del conocimiento no es una condición para el entendimiento del espacio, cada disciplina académica al construir modelos parciales, solo logra explicaciones limitadas, el abordaje debe ser multidisciplinario, cuyo método debe explicar las condiciones en las que opera el sistema global en el territorio, "se sabe muy poco en las ciencias sociales como para dividirse absurdamente" (Piketty, 2014: 48)

\section{El territorio desde el entendimiento disciplinar}

Las políticas de desarrollo en México, que se generaron en el siglo XX, en la búsqueda de integrar a las regiones más atrasadas al mercado, fueron diseñadas por la influencia de la teoría de la modernización que propone generar crecimiento económico a partir de procesos de intervención, tomando como elemento implícito las potencialidades del territorio (Tinbergen, 1989: 7). También como un sistema donde las mayores capacidades de una localidad pueden potenciar la región circundante a partir de un sistema de "polos de desarrollo" (Perroux, 1973: 10).

Estas propuestas impulsaron el desarrollo regional en un primer momento, pero fracasaron al agotarse el modelo del Estado de Bienestar a partir de la crisis de los años ochenta, que llevó a un cambio en los mecanismos de desarrollo, dando una preferencia a procesos basados en el mercado, limitando la intervención del gobierno, este giro hacia el "modelo neoliberal", provocó cambios en los procesos de intervención en el territorio, donde la base será la productividad del espacio y competitividad de los actores. 
El espacio como producto .... es el resultado del sistema social de alcance globalizado con su correlato de formas de dominación y sus efectos sobre esas y otras variables de alta respuesta en la organización del territorio... la modalidad del uso de los recursos naturales depende tanto de las necesidades y dinámica de la reproducción ampliada del capital (De Jong, 2001: 51).

Otro enfoque de análisis es la "geografía económica”, inicia con la explicación de "localización industrial”, que tiene a la empresa como el elemento básico del desarrollo económico en un territorio, este será ampliado con la "organización industrial”, que es la capacidad de interdependencia que existe entre la empresa con las demás empresas (la industria), con el territorio y sus encadenamientos productivos, estos modelos neomarshallianos comprenden además la capacidad de aprendizaje en el proceso (Sforzi, 2001, pp 20-22).

La "nueva geografía económica" (NGE), desarrollada por Krugman, le da un énfasis en lo territorial, en términos de factores físicos, procesos económicos y tecnológicos y en la creación de modelos de crecimiento económico, que genera una causación circular, donde los elementos de la aglomeración se auto refuerzan (Moncayo, 2003).

El "Desarrollo Local" entiende al territorio como un elemento indispensable en los procesos de organización económica, a partir de las potencialidades que allí existen, que las personas y organizaciones transforman y usan de forma racional y generan un sistema organizativo para dar respuestas a los retos que impone el desarrollo (Vazquez-Barquero, 2001: 48-50).

El “Desarrollo Sostenible” surge a partir de la preocupación por los efectos de degradación en el uso intensivo del medio natural, por el avance acelerado de un modelo basado en satisfacer un creciente consumismo, que permite una acumulación irrefrenable de las ganancias del capital, esta preocupación se ha mundializado, provocando políticas de alcance planetarias, integradas en los "Objetivos del Desarrollo Sostenible”(ODS) (Sachs, 2014).

Para el trabajo que se presenta el estudio del turismo ha tenido una importante aportación, a partir de la geografía del turismo (Piellet, 2015; Pinassi, 2015), de la planificación del espacio turístico (Vera, 1994; Linales y Morales, 2014; Miguel y Torres, 2014; Olivares, 2016) y desde la explicación de la estructura social (De-Uña y Villarino, 2011; Curiazi, 2014; Santana, 2015).

Las propuestas teóricas que se presentan sirven como un elemento indispensable para el entendimiento de la región de estudio, comprender las bases en las que se generaron las políticas públicas y ahora con visiones holísticas entender la realidad del territorio y de la sociedad allí asentada.

\section{Descripción demográfica y turística de la región}

Para la región de estudios los datos de población que ofrece el Instituto Nacional de Estadística, Geografía e informática (INEGI, 2018) y los del turismo generados por la Secretaria de Turismo de Jalisco (SECTURJAL 2016) permiten fundamentar la explicación del proceso histórico de la región.

La inexistencia de información de los primeros años de los municipios de Cabo Corrientes y la Huerta se debe a que fueron constituidos en la década de 1940, el primero era parte de Puerto Vallarta y el segundo de Casimiro Castillo y Tomatlán. El crecimiento en todos los municipios en la década de 1950, es explicado por el proceso de colonización de la costa, así como el despegue de Puerto Vallarta a partir de 1960 (Cuadro 1).

Cuadro 1: Crecimiento demográfico de los municipios de la costa de Jalisco (1920-2010)

\begin{tabular}{|l|c|c|r|r|r|r|r|r|r|r|}
\hline \multicolumn{1}{|c|}{ Municipio } & $\mathbf{1 9 2 0}$ & $\mathbf{1 9 3 0}$ & $\mathbf{1 9 4 0}$ & $\mathbf{1 9 5 0}$ & $\mathbf{1 9 6 0}$ & $\mathbf{1 9 7 0}$ & $\mathbf{1 8 8 0}$ & $\mathbf{1 9 9 0}$ & $\mathbf{2 0 0 0}$ & $\mathbf{2 0 1 0}$ \\
\hline Cabo Corrientes & & & & 4,364 & 5,077 & 5,068 & 6,785 & 8,146 & 9,133 & 10,029 \\
\hline Cihuatlán & 4,531 & 5,955 & 5,694 & 5,774 & 7,515 & 16,217 & 20,452 & 24,855 & 32,019 & 39,020 \\
\hline La Huerta & & & & 4,980 & 9,424 & 15,950 & 19,283 & 20,678 & 22,827 & 23,428 \\
\hline Tomatlán & 7,921 & 4,631 & 5,078 & 5,836 & 9,160 & 16,724 & 23,586 & 30,750 & 34,329 & 35,050 \\
\hline Puerto Vallarta & 4,574 & 10,245 & 10,471 & 10,801 & 15,462 & 35,911 & 57,028 & 111,457 & 184,728 & 255,681 \\
\hline
\end{tabular}

Fuente: elaboración propia a partir de datos de INEGI 2010 
Los datos obtenidos en los anuarios de la SECTURJAL, explican como se dio la trasformación de la región a partir del turismo, en Puerto Vallarta en la década de 1970, en tres años (1977- 1980) se duplican los turistas extranjeros y se triplican los nacionales (Cuadro 2)

Otro hecho tiene que ve con la sensibilidad que tuvo el turismo a la crisis estructural de la década de 1980 y la crisis global del 2008, que se manifiesta con una caída del turismo internacional.

A partir de 1990 la costa sur tiene un decrecimiento del turismo nacional por la baja calidad de la oferta en la región y un incremento del turismo extranjero por el impulso que se da en Careyes y Chamela.

\section{Cuadro 2: Afluencia Turística de la costa de Jalisco (1977-2016)}

\begin{tabular}{|c|c|c|c|c|c|c|c|c|c|c|}
\hline & & 1977 & 1980 & 1985 & 1990 & 1995 & 2000 & 2005 & 2010 & 2016 \\
\hline \multirow{2}{*}{$\begin{array}{c}\text { Puerto } \\
\text { Vallarta }\end{array}$} & Nacionales & 130,716 & 354,154 & 370,348 & 570,924 & 818,368 & 816,572 & $1,011,644$ & $1,052,934$ & $1,541,189$ \\
\cline { 2 - 11 } & Extranjeros & 234,116 & 517,997 & 269,383 & 628,161 & 646,162 & 942,083 & $1,057,029$ & 874,294 & 933,101 \\
\hline $\begin{array}{c}\text { Costa } \\
\text { Sur }\end{array}$ & Nacionales & 149,828 & 271,495 & 178,824 & 296,124 & 254,311 & 321,038 & 258,470 & 252,272 & 292,856 \\
\cline { 2 - 11 } & Extranjeros & 8,945 & 13,803 & 16,962 & 16,696 & 20,773 & 33,897 & 31,720 & 33,303 & 38,653 \\
\hline
\end{tabular}

Fuente: Elaboración propia a partir de datos de SECTURJAL 2016

\section{El poblamiento de la región a partir de procesos agropecuarios}

En la costa de Jalisco de 1850 a 1950 las actividades que se desarrollaron fueron agropecuarias con características de subsistencia y en menor medida la minería. Puerto Vallarta, nace mediados del siglo XIX a partir del desarrollo de actividades comerciales asociadas a las minas en la Sierra Occidental (Munguía (1977).

Para 1923 se dio una bonanza bananera, en la región de Puerto Vallarta, cuyo epicentro fue la población de Ixtapa, la compañía Montgomery, subsidiaria de la United Fruit Company, el producto era exportado por barco hacía Norteamérica (Goméz, 2003: 24-29; Andrade, 2006: 51).

Este proceso concluyó cuando el presidente Lázaro Cárdenas (1934-1940) inició el reparto agrario ejidal y las tierras que tenía la empresa fueron entregadas a los campesinos (Andrade, 2006: 52).

El Gobernador del estado Silvano Barba (1939-1943) propone el establecimiento de ejidos en toda la costa debido a la baja densidad poblacional, por la riqueza natural y para desarrollar proyectos agropecuarios que aportaran al mercado nacional en crecimiento (Rodríguez, 1989; Castillo, 1991; Luna, 1993).

El gobernador García Barragán (1943-1947) aceleró el proceso de colonización "es importante dejar el reparto agrario concluido en el centro y los altos de Jalisco ya saturados e iniciarlo en zonas semi pobladas como la costa" (Castillo , 1991: 101), los resultados no fueron los deseados por lo inhóspito del terreno, porque el Estado no contó con los recursos para realizar las obras de infraestructura y por los conflictos sociales que se generaron (Murià, 1982: 450).

El presidente Miguel Alemán (1946-1952) puso al turismo en el interés nacional, impulsando desde el gobierno federal al puerto de Acapulco, Guerrero ${ }^{1}$, como un destino de calidad mundial, al desarrollar proyectos de infraestructura, pasa de un puerto marítimo de carga a ser el primer destino de playa en el país.

En Jalisco el gobernador González Gallo (1947-1953), hará poco por la región costera, dedicando sus esfuerzos a la modernización industrial en Guadalajara. Por la trascedencia en el ámbito comercial y la generación de un turismo incipiente se construyen en el periodo de Vallarta los hoteles "Gutiérrez", "Rosita" y "Central" (Virgen, 2014: 30),

\section{El primer proceso de planeación de la costa}

El presidente Ruiz Cortines (1952-1958) desarrolló el "Programa de Progreso Marítimo", conocido como "la Marcha al Mar", será un elemento básico para el desarrollo económico y en la geopolítica nacional al ocupar amplias zonas de las costas (Tello, 2014a: 271). 
Esto llevó al gobernador Agustín Yáñez (1953-1959) a formar la "Comisión de Planeación de la Costa de Jalisco" (CPCJ)

La región de la costa se concibió como fuente de materias primas, campo potencial para inversiones altamente remunerativas y área de desahogo para las presiones demográficas... de satisfacción a la demanda de nuevos servicios turísticos, de aprovechamiento de áreas vírgenes (Álvarez, 1983: 88-89).

Yáñez dejó en su novela "La Tierra Prodiga" (1996), un relato de las condiciones que imperaban en la costa de Jalisco, los conflictos que se daban por la posesión del territorio, los esfuerzos por asentarse en una región agreste y la enorme belleza natural que se ofrecia entre la sierra y el oceano pacífico.

La comisión realizó varios proyectos y estudios, un centro turístico en la playa de Tenacatita, el primer censo de población en la región, estudio de las condiciones naturales regionales, apoyó los estudios para la explotación de la mina de manganeso en Autlán, "La comisión promovió, coordinó y ejecutó un buen número de obras de infraestructura en caminos, obras de irrigación, electrificación, salubridad, con la participación significativa de los pobladores de las localidades beneficiadas”. (Rodríguez, 1989: 16). Una obra fundamental era la conclusión del camino de Guadalajara- Barra de Navidad (proyecto que no se concluyó en ese tiempo).

A partir de un estudio de potencialidades turísticas en la costa, se planteó desarrollar tres zonas "Barra de Navidad-Tenacatita, Careyes- Chamela y Puerto Vallarta, asignándole ... potencial turístico, que se determinó por la accesibilidad y el atractivo ... 55\% a la primera, $20 \%$ a la segunda y $25 \%$ a la última" (Nuñez y Scartascini, 2010: 83), una acción que se desarrolló a partir de este estudio fue el proyecto de una ciudad moderna en Barra de Navidad con una zona turística y otra habitacional (Tello, 2014a: 286).

En Puerto Vallarta llega la empresa de Mexicana de Aviación y se elabora el plano regulador en 1955, que vendrán a perfilar a esa población como un polo de desarrollo (Virgen, 2014: 30).

En la década de 1950 a 1960 la población de los cinco municipios costeros creció en 46\% lo que demuestra la importancia del proyecto de la costa (Cuadro 1).

El gobernador del estado Gil Preciado (1959-1964), suprime el proyecto de la costa, motivado por la devastación que provocó el ciclón de 1959 que destruyó buena parte de la infraestructura regional, la salida de la Compañía Minera de Autlán al demostrarse que las minas de manganeso no eran rentables y era inviable la construcción del puerto industrial de Melaque y por la cancelación del proyecto de Barra de Navidad que era financiado por el empresario Ismael Madrigal quien se declaró en quiebra (Tello, 2014a: 287-289).

\section{La construcción de un destino en la costa norte y el proceso inconcluso en la costa sur}

En 1962, en la visita a Puerto Vallarta del expresidente Miguel Alemán, nombrado presidente del Consejo Nacional de Turismo, diversos actores sociales y políticos le mostraron las potencialidades turísticas del lugar, le expusieron la interminable construcción de las carreteras para conectar la costa con el centro del estado y la falta de procesos de comercialización de los productos agrícolas, este será uno de los hechos más trascendentes para su despegue.

En 1963 se filma en Puerto Vallarta, la película "La Noche de la Iguana” dirigida por John Huston, con la actuación de Richard Burton, Deborah Kerr y Ava Garner, tuvo varias nominaciones al Oscar (Montes de Oca, 2001: 266-268), se le recuerda más por las acciones mediáticas que se produjeron por la relación de pareja de Burton con Elizabeth Taylor, quienes construyeron casas y organizaron fiestas con invitados del Jet set internacional, aun hoy se le considera un elemento de promoción del destino a nivel global.

El gobernador Medina Ascencio (1965-1971) generó un nuevo impulso a la costa, reactivó la economía agrícola e impulsó el proyecto turístico de Puerto Vallarta, tres obras son emblemáticas, la conclusión de la carretera a Guadalajara, el puerto marítimo y el aeropuerto (en pocos años sera internacional). Se le nominó ciudad en 1968, trasformada en un centro vacacional moderno con la construcción de los hoteles "Posada Vallarta" (1964) y "Camino Real" (1969) y instalación del Banco Nacional de México. Otro hecho mediático fue la entrevista en la ciudad de los presidentes Nixon y Díaz Ordaz, en 1970 (Olvera, 1993; CEED- CEDESTUR, 2001).

El crecimiento poblacional de Puerto Vallarta en esa década fue del 123\%, lo que explica el despegue del destino, lo convierte en el principal polo de desarrollo de la región, el resto de la costa mantuvo una tasa de crecimiento similar a la década pasada (44\%) (Cuadro 1). No se cuenta con información sobre el 
comportamiento del turismo, pero se infiere que el crecimiento poblacional de Vallarta, la instalación de hoteles de cadena, la ampliación del aeropuerto y la carretera explican la aparición del turismo de masas.

En 1970 el presidente Días Ordaz (1964-1970), constituye el "Fideicomiso Bahía de Banderas" con la expropiación de 4,136 has. en Jalisco y Nayarit, su objetivo era el desarrollo turístico y habitacional de la región y la certidumbre en la propiedad para la inversión de proyectos turísticos.

En ese periodo se inició un programa de regionalización para la planeación, en la costa se constituyeron dos regiones, en el norte, con Puerto Vallarta, Cabo Corrientes y Tomatlán y en el sur, con Autlán, Purificación, Cuautitlán, Cihuatlán, Casimiro Castillo y la Huerta (CEED-CEDESTUR, 2001).

El presidente Echeverría (1970-1976) reimpulsó el reparto agrario en la costa (Rodríguez, 1989), generando conflictos en la región como el del ejido el Rebalsito de Apazulco, la dotación se da en tiempo record, pero más rápido se da la expropiación a favor del sindicato azucarero, para una vez construido su "Centro Vacional" se trasfiera a la empresa "Posadas de México" a mediados de los ochenta, esto se agravará por los conflictos por la posesión de espacios de playa entre ejidatarios e intereses privados encabezado por los descendientes del ex gobernador González Gallo (Ramírez, 1992: 73-76).

En 1971 se creó la Estación Biológica de Chamela de la Universidad Nacional Autónoma de México (UNAM), gracias a la donación del Dr. Antonio Urquiza de 1,600 has. y de 1,700 has. a la Secretaría de Agricultura (posteriormente las integrará a la estación bilógica) y 200 has. a la Universidad de Guadalajara (UdeG), la donación la realizó por el temor fundado de las expropiaciones ejidales (Tello, 2014b: 146-147).

En 1972 la conclusión de la carretera que une a Puerto Vallarta con Barra de Navidad, será un detonante para la realización de proyectos turísticos, pero provocó nuevos conflictos, al apropiarse empresas privadas de tierras ejidales para construir proyectos turísticos, como en la Boca de Tomatlán (Ramírez, 1992: 71).

En 1973 se constituye el "Fideicomiso Puerto Vallarta", cuyos objetivos eran "promover el desarrollo a través de la construcción y fraccionamiento, constituir empresas turísticas y negocios conexos y dar certidumbre jurídica a la propiedad de la tierra con la finalidad de que los inversionistas tuvieran seguridad legal de sus proyectos" (Scartascini y Nuñez, 2008: 173), esto dio origen a la zona hotelera norte de Vallarta que lo posicionara como un centro turístico mundial, con la operación de cadenas hoteleras internacionales.

La construcción de la presa Cajón de Peñas en el municipio de Tomatlán en 1974, permite el almacenaje de agua para irrigación, complementada con obras de canales de riego, este proyecto tenía como objetivo reimpulsar la actividad agropecuaria. (TPDS, 2012).

En la parte final del sexenio el reparto agrario se detiene, para dar certeza jurídica a la inversión privada en los nuevos proyectos, permitirá la consolidación del modelo de desarrollo en Careyes- Chamela, con la compra de tierras por inversionistas privados, para evadir la imposibilidad de propiedad en la costa por extranjeros, se constituyen fideicomisos (Avila y Luna, 2013: 68).

Tres proyectos impulsados por Gian Carlo Brignone explican este proceso de ocupación en la costa, el "Club Mediterrané" en 1972, en Playa Blanca, la construcción del "Hotel Plaza Careyes", financiado con la preventa de condominios y la construcción de la casa particular de Brignone, denominada "Mi Ojo", que determinara el estilo de construcción en la zona (Tello, 2014b: 57-70).

En 1978 el presidente López Portillo (1976-1982) creó la "Comisión de Conurbación del Río Ameca", entre Jalisco y Nayarit, buscaba el desarrollo urbano armónico, la coordinación en los servicios públicos, desarrollar proyectos productivos y el cuidado del medio ambiente (Gobierno de la República, 1978) esto se da por el despegue del proyecto "Nuevo Vallarta" en Nayarit y el despazamiento poblacional a localidades de ese estado.

En la década de 1970 el crecimiento de Vallarta fue del 58\%, no fue tan espectacular como la década anterior, es influida por la ampliación territorial hacía Nayarit, el crecimiento de Tomatlán (41\%) esta asociada a la construcción de la presa (Cuadro 1).

Puerto Vallarta tienen cerca de novecientos mil visitantes en 1980, (más de medio millón de extranjeros), que lo hace un importante destino turístico, en la costa sur el número de extranjeros esta determinado por los proyectos de Careyes-Chamela (Cuadro 2).

En los años ochenta, la construcción de la "Marina Vallarta" con infraestructura hotelera y marítima, desarrollada por el grupo SITUR, amplía la oferta turística e impulsa la construcción de departamentos de segunda residencia y busca captar al turismo náutico, se pretendía desarrollar una escalera náutica en el pacífico desde California EUA hasta Vallarta, proyecto que no se concluyó.

La crisis económica de los años ochenta impactara en la costa de Jalisco, en Careyes, "El Club Med", se encontraba por muchos periodos del año vacío (cerrara en los años noventa) y el hotel "Costa Careyes" estaba al borde de la quiebra, debido a las deudas acumuladas por su propietario. 
Brignone intentará reimpulsar su proyecto, en asociación con el empresario Gianni Pirri, generan el proyecto "Desarrollo Turístico de Costa Careyes", era un desarrollo inmobiliario, una marina (Puerto Careyes) y un campo de golf, generó interés por parte de inversionistas internacionales (Trafalgar House Construcción Holding Limited, Barclays Bank y los Pritzker, dueños de la cadena Hyatt) (Tello, 2014b: 72-73), el proyecto no se concreta por los efectos de la crisis económica y la intervención de James Goldsmith, quien opera para evitarlo, pues había comprado grandes extensiones en Cuixmala, vecina del proyecto, donde proyectaba construir su casa (Tello, 2014b: 112), este será el inicio de una lucha entre dos visiones, "proteccionistas" y "desarrollistas".

El presidente Miguel de la Madrid (1982-1988) orientará su gobierno a la estabilización económica posterior a la crisis, implementando el modelo neoliberal, además en 1985 el terremoto que causo graves daños a la infraestructura y vivienda en la ciudad de México, obligará a reorientar el gasto público hacía la reconstrucción.

\section{La Costalegre y la conformación de la reserva de la biosfera}

El gobernador del estado Guillermo Cosío (1989-1992) presentó el "Plan Sistema Jalisco XAPAC XXI", era un proyecto integral para la costa, trataba de

consolidar como polos de desarrollo de Puerto Vallarta y Barra de Navidad... el equilibrio, bienestar social y económico de los asentamientos humanos... aprovechar racionalmente los recursos naturales y la conservación del equilibrio ecológico... planteaba un aeropuerto en la Costalegre y la ampliación a cuatro carrieles de la carretera 200 (Tello, 2014b: 106).

El gobierno de la República bajo la presidencia de Carlos Salinas (1988-1994) presenta "La declaratoria de zona de desarrollo turístico prioritario a la zona de Costalegre", con una dotación de 577.2 has. la Secretaria de Desarrollo Urbano, el gobierno de Jalisco y los ayuntamientos instrumentarían la declaratoria de uso de suelo turístico y la secretaria de Turismo apoyaría la creación de empresas turísticas, gestionaría financiamiento para el desarrollo de la región y realizaría proyectos ejecutivos (DOF, 1990).

A partir ambos proyectos se generó un "Plan para el Desarrollo Regional de la Costa de Jalisco", que integraba los planes maestros, Gran Bahía de Chamela (propuesto por el grupo SITUR), ordenamiento ecológico de la costa de Jalisco, proyecto de puerto pesquero y turístico de Punta Pérula, fraccionamiento turístico en Cajón de Peñas, centro de usos múltiples de Puerto Vallarta, puerto peatonal costero de Puerto Vallarta, remodelación del malecón de Barra de Navidad y remodelación urbana de la Manzanilla (César, 2007: 149-153).

A finales de la década de 1980 la familia Brignone, propuso varios proyectos de baja densidad en Careyes, en los humedales de Punta Farallón y el cerro de la Cal (Tello, 2013: 224), estos no fueron ejecutados, por la situación financiera del grupo, que obligó a que muchas de sus propiedades fueron vendidas para pagar deudas acumuladas (Tello, 2014b: 104-105).

En la década de los ochenta, Puerto Vallarta tiene un crecimiento poblacional del 95\% que contrasta con el resto de la costa que sólo crece al 20\% (Cuadro 1). Puerto Vallarta tuvo una afluencia de más de un millón doscientos mil turistas, en la costa sur el número de turistas fue similar al de diez años antes, esto motivó los proyectos de intervención en la región (Cuadro 2).

Goldsmith constituye en 1988 la "Fundación Ecológica Cuixmala A. C", su objetivo

llevar a cabo todo tipo de actividades relacionadas con la preservación y restauración del equilibrio ecológico... promover ante las autoridades competentes el decretar y/o emitir disposiciones legales que tengan por objeto la protección del sistema ecológico ... y promover y llevar a cabo estudios e investigaciones tendientes a mejorar el conocimiento del sistema ecológico del estado de Jalisco (Tello, 2013: 222)

En 1990 la "Fundación Ecológica" y la estación biológica de la UNAM terminaron el estudio para crear la "Reserva de la Biosfera de la Costa de Jalisco", en la región de Cuixmala- Chamela (UNAM, 2018) su importancia desde el punto de vista biológico es descrito por Castillo Et Al. (2009)

El bosque tropical seco (BTS) cubre una importante superficie de la costa occidental de México... se reconoce como un sitio de extrema importancia para la conservación debido al alto número de especies (1200 de plantas y 422 de vertebrados) y a que muchas de éstas ( $40 \%$ de plantas y $14 \%$ de aves y mamíferos) son endémicas (844) 
Existen dos motivaciones de Goldsmith, aunque no contradictorias, si muestran un doble discurso, el empeño de la conservación y el gusto porque no haya nada que quite la vista de la zona natural que rodea su propiedad (residencias para su familia e invitados y extensiones donde llevará a vivir animales exóticos).

Tres hechos exógenos suceden en 1992 incidirán en la costa de Jalisco, la reforma del artículo 27 constitucional (que permite que los ejidos puedan desincorporar tierras para venderlas a particulares), las explosiones del drenaje de Guadalajara del 22 de abril y la posterior destitución del Gobernador Cosío (hecho frenara proyectos que impulsaba su gobierno en la costa) y la "Cumbre de la Tierra" de Río de Janeiro en junio.

El Desarrollo Sostenible será una de las principales estrategias del gobierno, a partir de que las naciones desarrolladas pusieron un interés particular por el tema y lo integraron como un elemento en los proyectos comunes, como en las negociaciones del Tratado de Libre Comercio de América del Norte (TLCAN), donde se integró como un elemento marginal, pero importante, para el grupo gobernante, donde se dio un cambio generacional, era una buena oportunidad por su interés de ser protagonista en el escenario internacional y apoyar las acciones de la ONU les resulto atractivo y porque grupos de presión internos particularmente provenientes de la investigación en las universidades lo pusieron como un asunto que debería ser incluido en los planes de desarrollo del país.

Una estrategia que surge de este interés fue la constitución de la Comisión Nacional para el Conocimiento y Uso de la Biodiversidad (CONABIO), su función será la declaratoria de áreas naturales protegidas, una de las decisiones más controvertidas, por cómo sucedieron los hechos fue la declaratoria de la Reserva de la Biosfera Chamela-Cuixmala.

El grupo encabezado por Goldsmith presionará para su aprobación con la amenaza de publicar desplegados donde evidenciaba el nulo interés por el medio ambiente del gobierno, con el apoyo de importantes personajes internacionales, como los ex presidentes de EUA Reagan y Carter, los ex primer ministros del Reino Unido, Tatcher y Wilson, el investigador Jaques Cousteau, Maurice Strong, secretario general del PNUMA, representantes de medio de comunicación como Rupert Murdoch, Conrad Black y miembros de la realeza europea, entre otros más (Tello, 2014b:167-168).

Situación que afectaba los intentos de protagonismo del grupo gobernante y que resultaba crítico, para las negociaciones del TLCAN, que tenía como elemento de preocupación colateral la protección ambiental, Goldsmith usará sus influencias para conseguir votos en el congreso norteamericano, que los usará según sus intereses (Tello, 2013: 226). Después de muchas negociaciones el gobierno aprueba en diciembre de 1993 la declaratoria de reserva de la Biosfera, el manifiesto propuesto se publica, pero como una expresión de apoyo a la acción desarrollada en favor del medio ambiente y en enero de 1994 entra en vigor el TLCAN.

El grupo "desarrollista" encabezado por Brignone tuvo que ceder, pudiendo conservar terrenos que no se encontraban dentro del polígono de la reserva y por negociación desde el gobierno para que no perdiera todas las opciones de inversión. A pesar de la declaratoria de la Reserva, persisten conflictos con los campesinos, debido a los perjuicios económicos que les provocó dicha resolución, por no poder usar las tierras que quedaron dentro o cerca de la reserva (Castillo, et al, 2006: 97).

La crisis económica de 1994 provocará que el grupo SITUR, constructor de Marina Vallarta y promotor de la costa de Chamela, entre en quiebra, será rescatado por el gobierno a través del Fondo Bancario de Protección al Ahorro (FOBAPROA).

El presidente Ernesto Zedillo (1994-2000) recibió del gobierno de Japón un estudio para la "Formulación de estrategias de desarrollo y promoción para destinos turísticos seleccionados de México" (JICA, 1997) que incluía a Los Cabos, Puerto Vallarta y Cancún, describe la sobre-dependencia de un solo segmento de mercado, la deficiente adaptación a las diversificadas necesidades del consumidor, la inexistencia de un sistema de desarrollo integrado, la inadecuada colaboración entre destinos, la marginación de la población local del desarrollo turístico, la cultura desechable del turismo, las amenazas a las áreas naturales, la deficiente competitividad de los servicios turísticos y la necesidad de descentralizar la administración turística.

En el caso de las estrategias proponía impulsar el triángulo turístico de Puerto Vallarta- Guadalajara y la Costalegre, a partir de la conservación y embellecimiento del centro histórico de Puerto Vallarta, el desarrollo del complejo turístico en la ladera de la montaña, el desarrollo del centro de convenciones, la generación de identidad de poblado mexicano en Guadalajara, el mejoramiento de las amenidades en Tequila, San Sebastián, Talpa y Mascota y la conclusión de la carretera Puerto Vallarta-Mascota.

En enero de 1999 se aprobó el "Ordenamiento ecológico territorial de la zona de Costalegre", que afianzó y legitimó las políticas de protección en la zona, pero también abrió "candados" para el desarrollo de proyectos turísticos (Avila y Luna, 2013: 74). 
En cuanto al turismo, Vallarta mantuvo un crecimiento importante, con un millón setecientos cincuenta mil visitantes (el 53\% extranjeros), en la costa sur aunque hay un incremento en el número de turistas extranjeros, en términos reales es muy bajo y el turismo nacional tampoco tuvo un crecimiento significativo (Cuadro 2).

\section{Los nuevos procesos en la costa de Jalisco en el nuevo siglo}

Un grupo de empresarios financió conjuntamente con la Universidad de Guadalajara el estudio "Bahía de Banderas a Futuro", evaluó la región que comprende Cabo Corrientes y Vallarta en Jalisco y Bahía de Banderas en Nayarit. Propuso un plan estratégico para los procesos de planeación y para la solución a los problemas comunes, se destaca que el turismo es la actividad básica en que funciona la región y se debe apoyar un proceso de diversificación económica (CEED- CEDESTUR, 2001). La UdeG, a partir del proceso de formación de la Red Universitaria en 1994 constituyó centros regionales como lo es el de la Costa, con sede en Vallarta, ofreciendo programas académicos e incidiendo en el desarrollo regional de las zonas de influencia, a partir de procesos de investigación y vinculación.

En el 2002, la UdeG, desarrolló el proyecto de "Vocacionalidad turística de la zona costera de Jalisco", financiado por el gobierno del estado, su objetivo era el descubrimiento de áreas con potencialidad a ser desarrolladas a partir del turismo (César y Arnaiz, 2004).

En el año de 2004, presentó la Secretaría de turismo federal el "Diagnóstico del sistema de indicadores de sustentabilidad de turismo de Puerto Vallarta" (SECTUR, 2004), a partir de la Agenda local 21, el resultado es positivo en los indicadores cualitativos y regular en los cuantitativos, pues se demuestra que no se cuenta con información para evaluar muchos indicadores.

Al final de la presidencia de Vicente Fox (2000-2006), se autorizaron dos proyectos en la costa de Jalisco, el "IEL" conocido como "Marina Careyes", y "La Tambora", proyectaban la construccción de más de mil habitaciones hoteleras, un campo de golf y una marina para 160 yates. La UNAM y la Fundación Cuixmala, presentaron una solicitud de reconsideración, señalando las serias amenzas a las reservas de agua y los humedales en la Reserva de la Bisofera, logrando parar los proyectos (Tello, 2013; Avila y Luna, 2013).

En la presidencia de Felipe Calderon (2006-2012), se autorizá la manifestación de impacto ambiental del proyecto denominado Zafiro (MIA-Zafiro, 2010), se localiza en la costa de Chamela, con una extensión de 910 has., se proyectan áreas residenciales, un hotel, restaurantes, comercios, clubes de playa, a pesar de contravenir el "Ordenamiento ecológico Territorial de la costa de Jalisco" (Hernández, 2010; Avila y Luna, 2013; Castillo, et al. 2006).

Este proyecto se hizo sin la aprobación de actores locales (pescadores y habitantes de las comunidades vecinas), que se agrava con el asesinato del lider de la Unión de pescadores de la Costa Sur de Jalisco, Aureliano Sánchez en 2011, quien defendía el acceso a la playa y los humedales aledaños a la desembocadura del río Cuixmala y el acceso a la playa Careyitos (Avila y Luna, 2013:76).

La población de Puerto Vallarta creció al 38\%, el porcentaje más bajo los últimos 50 años, el resto de los municipios no tuvieron diferencias a las condiciones anteriores (Cuadro 1).

La baja en el número de turistas extranjeros en el año 2010 está determinada por la crisis global de 2008, el turismo nacional tuvo un crecimiento importante por las mejores carreteras y una reorientación de la mercadotecnia hacía ese segmento. En la costa sur, la situación se mantiene en un nivel de poco crecimiento, donde el turismo nacional tuvo una contracción importante (Cuadro 2).

Los últimos años se presentaron proyectos similares para la reactivación de Puerto Vallarta financiados por el FONATUR, en 2010 se presentó "Líneas de acción para el ordenamiento turístico del Centro histórico de Puerto Vallarta" (SECTURJAL-FONATUR, 2010), se basa en un diagnóstico de su problemática, agravada por los efectos que provocó el huracán Kenna del 2002, plantea hacer una trasformación integral, basada en la imagen urbana, los espacios públicos, el patrimonio, la vialidad y el transporte y el rescate del patrimonio.

En 2012 se presenta el "Programa de desarrollo turístico del centro histórico de Puerto Vallarta, Jalisco", (SECTURJAL-FONATUR, 2012), tiene la característica de dejar fuera del proyecto al malecón de Puerto Vallarta, porque se había iniciado la intervención con recursos federales dado el nivel de deterioro y la urgencia por los graves daños estructurales. Retoma parte del proyecto de 2010, agregando la falta de atractivos turísticos, baja oferta hotelera y de vivienda vacacional, y riesgos ambientales. Presenta como proyectos detonantes: complejo metropolitano de deporte, recreación y cultura, plaza 
del mar (parque Hidalgo), museo arqueológico y natural del río Cuale, parque escultórico y anfiteatro (parque Lázaro Cárdenas) y revitalización del distrito Camarones para vivienda de segunda residencia.

En 2012 por iniciativa de un grupo de empresarios se realiza el "Plan maestro de la zona romántica de Puerto Vallarta Jalisco" (Estudio 3.14, 2011), tiene el objetivo de reactivar las actividades comerciales y turísticas, se basa en la creación de un esquema de autenticidad e identidad propios, la creación de un espacio confortable y seguro y la conectividad con otras zonas del destino.

En 2015 la secretaria de turismo federal del gobierno de Enrique Peña (2012-2018) solicitó a diversas Universidades la realización de "Agendas de Competitividad Turística", para Puerto Vallarta se realizó a partir de un diagnóstico y el alineamiento de los diversos ordenamientos de política pública en materia de turismo (SECTUR-UdeG, 2013), presentando proyectos detonantes: usando las que había propuesto FONATUR en 2010 y agregando un teleférico de la zona de montaña al centro, trasporte troncal de calidad turística, un tipo tranvía que atraviese el centro histórico, corredor ecoturístico en el vecino Cabo Corrientes, la trasformación del barrio Gringo Gulch, un Acuario y museo oceanográfico, rehabilitación del Boulevard Medina Ascencio y rehabilitación urbana del centro histórico.

En 2010 la Administración Portuaria Integral de Puerto Vallarta presentó el "Programa maestro de desarrollo portuario de Puerto Vallarta" (API, 2010), es un estudio sobre las potencialidades del puerto, con el desarrollo de infraestructura básica, acciones a favor del medio ambiente y la seguridad para los cruceros y las embarcaciones locales.

En 2011 presentó el "Plan de competitividad de Puerto Vallarta como destino de cruceros" (API, 2011) su objeto es posicionar al destino dentro de los principales lugares de visita de crucero, porque es el puerto del pacífico medio más visitado, donde se realizan el mayor número de actividades asociadas a este segmento. Se presenta un portafolio de productos que afirmen la identidad del destino, acciones de infraestructura, generación de procesos de competitividad, mercadotecnia y seguridad. Para resaltar la importancia de este segmento, en el año 2017 se recibieron 145 cruceros con 333,153 visitantes (Dirección General Puertos S. C. T., 2017).

En 2011 el gobernador Emilio González (2006-2012), presentó el "Programa subregional de desarrollo turístico de Costalegre, Jalisco" (SECTURJAL- FONATUR, 2011) financiado por FONATUR, con una superficie de 71,122 Kms. cuadrados y un litoral de $311 \mathrm{Kms}$. de longitud, presenta un diagnóstico de la actividad turística en general y para cada municipio y un portafolio de doce proyectos estratégicos para relanzar atractivos de la región a partir de su perfil y sus potencialidades, que incluye un proyecto comercialización, gestión y monitoreo.

Otro conflicto se da en el "Hotelito Desconocido" en la costa de Tomatlán, después de gozar una buena fama como un proyecto sostenible y con buenas relaciones con las comunidades vecinas, esto cambio en los útimos años, en marzo de 2011 fueron secuestrados en Guadalajara tres pescadores, llevaban tiempo oponiéndose a la destrucción de manglares debido al dragado del estero el Ermitaño para construir un canal y ampliar la extensión del hotel (Avila y Luna, 2013: 80), en el año 2015 la PGR asegura el negocio por la presunción de ser propiedad de la delincuencia organizada. (Debate, 2016).

En el año 2012, la UdeG desarrolló el estudio prospectivo “Tomatlán a futuro" (Espinoza, et al. 2015), presenta proyectos estratégicos de desarrollo urbano, turismo, generación de empresas, educación, cultura, salud y cuidado del medio natural, además de una cartera de obras de infraestructura básica para fortalecer las capacidades del municipio.

En 2015 inicia la construcción del proyecto Zafiro, se construyen los hoteles Cheval Blanc (52 habitaciones y el restaurant "LE 1947"), Four Season (160 habitaciones) y One and Only (75 villas), un campo de golf de 18 hoyos y una marina de yates (Huerta, 2016), un compromiso del gobierno complementario al proyecto es la construcción de una aeropista con capacidad de recibir aviones ejecutivos.

Ese mismo año se presentó la Manifestación de Impacto Ambiental, para el "Desarrollo Vistas Chalacatepec", en el polígono del proyecto se localizan dos sitios RAMSAR, las lagunas Chalacatepec y la Paramán-Xola, el proyecto tiene una extensión de cerca de 1,200 has. donde se tiene proyectado un conjunto inmobiliario y hotelero, un área natural protegida de 515 has. y la reforestación de 239 has. se tiene avanzada la construcción de un aeropuerto (Entorno Turístico, 2015), la empresa desarrolladora "Rasaland", esta asociada con el "Instituto de Pensiones del Estado de Jalisco" (IPEJAL, 2015), que es el administrador de los fondos pensiones de los trabajadores del gobierno, situación que generó muchas suspicacias mediáticas. Existen litigios promovidos por grupos de campesinos que no están de acuerdo con las formas como se realizaron las compras de las tierras (Del Castillo, 2015).

En 2017 se concluyó la primera parte de la autopista de Jala, Nayarit- Puerto Vallarta Jal. Con el tramo Jala- Compostela y se iniciaron las obras de modernización del recinto portuario de Puerto Vallarta, con la construcción de una terminal de pasajeros, estacionamiento y adecuaciones de los 
espacios de atraque de cruceros y embarcaciones locales, dentro del recinto portuario se construye un acuario por la empresa "Blue Life", será uno de los tres más grandes de país, contará con más de 300 especies marinas (Entorno turístico, 2017).

Ese mismo año el gobierno del estado encabezado por Aristóteles Sandoval (2012-2018) realizó el "Plan Maestro y Estratégico de Turismo Incluyente en la Costa de Jalisco", que da sustento a la primera playa con accesibilidad universal en Cuastecomates en el municipio de Cihuatlán. (Gob. Jal., 2017)

\section{Conclusiones}

El proceso de poblamiento en la costa de Jalisco, se da a partir de las políticas públicas, determinadas por las orientaciones teóricas de la modernización en dos momentos, primero al dotar de terrenos ejidales a campesinos para el desarrollo de proyectos agrícolas, pero también como un proceso de geopolítica para ocupar espacios vacíos del territorio, por la necesidad de control por la nueva configuración política internacional.

En el siguiente proceso de poblamiento, utiliza como estrategia al turismo, que reconfigura la estructura social y modifica de manera importante al paisaje al ser apropiado como una mercancía, inicia de manera importante a finales de los años sesenta, provocando desequilibrios intraregionales, al desarrollar de forma intensiva a Puerto Vallarta y de menor medida la zona de Chamela, dejando al resto de la costa con una infraestructura que se convertirá en obsoleta y que no habrá mecanismos para su revitalización, particularmente en Melaque- Barra de Navidad.

El turismo de masas explica el crecimiento de Puerto Vallarta en la década de 1970, el reimpulso a partir de la construcción de la Marina Vallarta en la década de 1980, pero también efectos negativos por las crisis económicas recurrentes, después de una década de la crisis global del 2008, el turismo aun no recupera los niveles previos, aunque la población no dejo de crecer en ese periodo.

A partir de que el turismo se apropia del espacio y las comunidades asentadas en el territorio se involucran en la actividad turística, se modifica las formas de vida de la población generando efectos positivos, como empleo y ampliación del mercado interno, pero también efectos negativos, los conflictos por la propiedad de la tierra, perder accesos a espacios que antes eran públicos y ahora se han privatizado, deja a la población, en muchos casos, fuera de los beneficios prometidos.

El caso extremo y mejor documentado es Chamela- Careyes, con cotos cerrados, rodeada por un área natural protegida que les da mayor privacidad y valor, y ahora está siendo reimpulsada con la construcción de nuevos proyectos similares.

La ocupación del espacio genera conflictos entre los diversos actores, cada uno defenderá su posición, de ello depende su patrimonio e inversiones, haciendo una caracterización a partir del relato propuesto, se pueden denominar los grupos como "productivistas" quienes dependen de la actividad agrícola, "desarrollistas" quienes generan proyectos de inversión para la actividad turística, los "proteccionistas", quienes luchan por mantener o recuperar los equilibrios ambientales en una región mega diversa, y una población "desposeída", que ha migrado para trabajar en la construcción y que se asienta en el territorio dependiendo de las pocas oportunidades que se presentan.

Estos grupos interactúan en el territorio, interviniendo desde su posición, pero con ello provocan conflictos que perduran en el tiempo y cuya solución es compleja. Se requiere una enorme voluntad que concierte los diversos esfuerzos, se debe potenciar el beneficio de la sociedad y al cuidado del medio natural.

La configuración de la región, depende de las políticas públicas, que fomentaron el desarrollo con obras de infraestructura y procesos para impulsar las actividades productivas, generando enormes avances a pesar de lo agreste del terreno y la inexistencia de proyectos, la no continuidad en el largo plazo de los proyectos, es una constante que no permitió mejores resultados.

Este trabajo sirve como un primer intento de construir una línea del tiempo que permita contar con un hilo conductor de la realidad de la costa. Reunir aquí los proyectos documentados que sirva como un elemento base para continuar con la investigación de la costa es un objetivo de largo plazo.

\section{Bibliografía}

Álvarez, José Rogelio.

1983. "La comisión de planeación de la costa de Jalisco". Revista Encuentro, 1. Oct.- Dic. Andrade, Manuel.

2006. “Tiempos inolvidables de Puerto Vallarta”. México. Ed. Universidad de Guadalajara. 
API. (Administración Portuaria integral de Puerto Vallarta).

2010. "Programa maestro de desarrollo portuario de Puerto Vallarta". Disponible en https://www. puertodevallarta.com.mx/Documentos/PMDP_Vallarta_2010-2015.pdf

API. (Administración Portuaria integral de Puerto Vallarta).

2011. "Plan de competitividad de Puerto Vallarta como destino de cruceros". Disponible en https://secturjal. jalisco.gob.mx/sites/secturjal.jalisco.gob.mx/files/u16/07_plan_de_competitividad_cruceros_p._vallarta.pdf Avila, Patricia y Luna, Eduardo.

2013. "Del ecologismo de los ricos al ecologismo de los pobres". Revista Mexicana de Sociología.

Castillo, Alicia; Pujadas, Anna; Magaña, María y Godinez, Carmen.

2006. "Comunicación para la conservación: análisis y propuestas para la reserva de la biosfera Chamela-

-Cuixmala, Jalisco". En Burahonda, Ana y Almeida, Lucia. "Educación para la consevación”. México.

Ed. Las prensas de ciencias UNAM.

Castillo, Alicia; Schroeder, Natalia; Galicia, Claudia; Pujadas, Anna y Martínez, Lucia.

2009. "El bosque tropical seco en riezgo: conflictos entre uso agropecuario, desarrollo turístico y previsión de servicios ecositémicos en la costa de Jalisco”. México. Revista Interciencia, 34 (12).

Castillo, Carlos.

1991. "El proyecto de colonización de la costa de Jalisco: primera etapa 1944-1947”. Revista de Estudios Regionales.

CEED-CEDESTUR.

2001. "Bahía de Banderas a futuro: construyendo el porvenir 2000-2025”. México. Ed. Universidad de Guadalajara.

César, Alfredo y Arnaiz, Stella.

2004. "Desarrollo y turismo en la costa de Jalisco". México. Ed. Universidad de Guadalajara.

César, Fernanda.

2007. "Ciudades Turísicas: desarrollo e imaginarios Careyes y Nuevo Vallarta”. México. Ed. Universidad de Guadalajara.

Curiazi, Roberta.

2014. "Diálogo social y cooperación territorial en los destinos turísticos: los "buenas prácticas" para la sostenibilidad”. Revista TURyDES Revista de investigación en turismo y desarrollo local, 7(16).

Debate.

2016. El "Hotelito Desconocido" un paraiso del narco. 16 de marzo de 2016. Recuperado el 20 de 09 de 2017, disponible en https://www.debate.com.mx/mexico/El-Hotelito-Desconocido-el-paraiso-del-narco-20161203-0142.html

De. Jong, Mario

2001. "Introducción al método regional". Argentina. Ed. LIPAT Univ. Nacional del Comahue.

Del Castillo, Agustín.

2015. "Chalacatepec ya tiene proyecto ambiental". Periodico Milenio, 9 de julio de 2015.

De Uña, Elena y Villarino, Montserrat.

2011. "Configuraciones de identidad en territorios del turismo: Consideraciones generales de Galicia". Revista Cuadernos de Turismo (27): 259-272.

Dirección General de Puertos S. C. T.

2017. Cruceros atendidos 2017. Disponible en http://www.sct.gob.mx/fileadmin/CGPMM/U_DGP/ estadisticas/2017/Mensuales/12_diciembre_2017.pdf

DOF (Diario Ofical de la Federación).

1990. "Declaratoria de Zona de Desarrollo Turístico Prioritario". Ed. Diario Oficial de la Federación 5 de dic. de 1990.

Entorno Turístico.

2015. "Chalacatepec, el nuevo Cancún". Recuperado el 13 de 09 de 2017. Disponible en http://www. entornoturistico.com/chalacatepec-el-nuevo-cancun/

Entorno turístico.

2017. "Nuevo acuario en Puerto Vallarta. Entorno turístico". Recuperado el 13 de 09 de 2017. Disponible en https://www.entornoturistico.com/nuevo-acuario-de-puerto-vallarta/

Espinoza, Rodrigo; Tellez, Jorge; Chavez, Rosa; Andrade, Edmundo y Cornejo, José Luis.

2015. "Tomatlán a futuro, edificando el porvenir 2012-2040". México. Ed. Universidad de Guadalajara. Estudio 3.14.

2011. "Plan estrategico zona romantica de Puerto Vallarta". Disponible en http://secturjal.jalisco.gob. $\mathrm{mx} /$ desarrollo-turistico-regional 
Gobierno de la República.

1978. "Plan de ordenamiento de la zona conurbada del río Ameca". Gobierno de la República, México.

Documento impreso.

Gob. Jal.

2017. "Plan maestro y estratégico turismo incluyente en la costa de Jalisco". DIF, SIOP Gobierno del

Estado de Jalisco, México. Documento impreso.

Goméz, Eduardo.

2003. "Ixtapa, entre el ensueño y el isomnio, la sociedad mercantio Montgomeryand Cia. en la región de

Bahía de Banderas 1924.1935”. México, Ed. Planeta.

Hernández, Sergio.

2010. "Proyecto Zafiro en Chamela. Verdevandera". periodismo ambiental. México. 15 de sept. 2010.

Huerta, Juan.

2016. "Louis Buitton contruye hotel en Jalisco". Periodico El financiero, 2 de enero de 2016. México.

INEGI I(Instituto Nacional de Estadítica, Geografía e Informática)

2018. Censo de Población y vivienda. Disponible en http://www.inegi.org.mx.

IPEJAL. (Instituto de Pensiones del Estado de Jalisco)

2015). ¿Qué es Chalacatepec? Recuperado el 13 de 09 de 2017, Disponible en http://www.ipejal.gob.mx/

Chalacatepec/index.html

JICA. (Agencia de Cooperación Internacional de Japón).

1997. "México, Estudio para la formulación de estrategias de desarrollo y promoción para destinos turísticos seleccionados en México”. Recuperado el 14 de 09 de 2017, Disponible en http://open_jicareport.jica. go.jp/pdf/11352572_01.pdf

Linares, Hermys y Morales, Geily.

2014. "Del desarrollo turístico sostenible al desarrollo local, su comportamiento complejo". Revista Pasos, 12 (2): 453-466.

Luna, Pablo.

1993. "Vallarta y su región durante la primera mitad del siglo XX". En Olvera Jaime, Una aproximación a Puerto Vallarta. México: Ed. El Colegio de Jalisco.

MIA-Zafiro.

2010. "Plan de ordeanmiento Ecológico MIA Zafiro". Verdevandera, periodismo ambiental. Recuperado el

20 de 09 de 2017, Disponible en http://verdebandera.com.mx/wp-content/uploads/2015/01/Resolutivo-

-MIA-Zafiro 14JA2009T0017.pdf

Miguel, Andrés y Torres, Julio.

2014. "El impacto territorial del turismo en el desarrollo sostenible: el caso de las regiones de México 2000-2010”. Revista Pasos, 12(2): 357-368.

Moncayo, Edgard.

2003. Nuevas teorías y enfoques conceptuales del desarrollo regional ¿Hacía un nuevo paradigma?

Revista de Economía Institucional, 8(5): 32-65.

Montes de Oca, Catalina.

2001. "Puerto Vallarta, en mis recuerdos". (2 $2^{a}$ edición ed.). México. Ed. Universidad de Guadalajara.

Munguia, Carlos.

1977. "Panorama histórico de Puerto Vallarta (1800-1918)". En Olvera, Jaime Una aproximación a

Puerto Vallarta. México. Ed. Colegio de Jalisco.

Murià, José. María.

1982. Historia de Jalisco (Vol. 4). México. Editorial UMED.

Nuñez, Patricia y Scartascini, Gabriela.

2010. "Política económica y desarrollo regional: La costa de Jalisco a mediados del siglo XX." En

Nuñez, Patricia y Arnaiz Stella. "Impactos y dimenciones del turismo". México. Ed. Universidad de Guadalajara: 65-86.

Olivares, Adriana.

2016. "La gestión de los territorios turísticos en América Latina". Revista ACE: Arquitecture, city aln

Envioronment, 11(31).

Olvera, Jaime.

1993. "Una aproximación a Puerto Vallarta”. México. Ed. El Colegio de Jalisco.

Perroux, Francoix.

1973. "Nota sobre el concepto de polo de crecimiento". Argentina. Ediciones Nueva Visión. 
Piellet, Felix.

2015. "Del espacio geográfico al turismo como uso y disfrute del territorio comarcal: una reflexión teórica desde España”. Revista de Geografía Norte Grande 62: 185-2010.

Pinassi, Andrés.

2015. "Espacio vivido; análisis del concepto y vínculo con la geografía del turismo". Revista Geógrafos, 6(78).

Piketty, Thomas.

2014. "El Capital en el siglo XXI". México. Ed. F. C. E.

Ramírez, Luis.

1992. "Fuego en el paraíso: turismo y conflictos en las tierras prodigas". Revista Relaciones (50).

Rodríguez, María.

1989. "Población y poblamiento en la costa de Jalisco". Revista de Estudios Sociales (6).

Sachs, Jeffrey.

2014. La era del desarrollo sostenible. España. Ed. DEUSTO.

Santana, Agustín.

2015. "Imaginando la imagen en turismo: un viaje de ida y vuelta". Revista de Antropología Experimental (15): $37-53$.

SECTUR. (Secretaría de Turismo)

(2004). Agenda local 21 de Puerto Vallarta, Sistema de indicadores de sustentabilidad para el turismo. México. Secretaría de Turismo.

SECTURJAL-FONATUR. Secretaria de Turismo de Jalisco- Fondo Nacional de Turismo.

2010. "Líneas de acción para el ordenamiento turístico del Centro histórico de Puerto Vallarta,

Jalisco-Disponible en: https://secturjal.jalisco.gob.mx/sites/secturjal.jalisco.gob.mx/files/u16/06_lineas_de_accion_para_el_ord_turist._de_pv_presentacion_ejva.pdf

SECTURJAL.

Anuario Estadísitico 2016. Disponible en https://secturjal.jalisco.gob.mx/invierte-en-jalisco/estadisticas.

SECTURJAL- FONATUR. Secretaria de Turismo de Jalisco- Fondo Nacional de Turismo.

2011. "Programa subregional de desarrollo turístico de Costalegre, Jalisco." Disponible en: https:// secturjal.jalisco.gob.mx/sites/secturjal.jalisco.gob.mx/files/u16/08_costalegre_presentacion_ejecutiva.pdf

SECTURJAL-FONATUR. Secretaria de Turismo de Jalisco- Fondo Nacional de Turismo.

2012. "Programa de desarrollo turístico del centro histórico de Puerto Vallarta, Jalisco". Disponible en: https://secturjal.jalisco.gob.mx/sites/secturjal.jalisco.gob.mx/files/u16/09_pdt_centro_historico_pv_presentacion_ejecutiva.pdf

SECTUR-UdeG. Secretaria de Turismo. Universidad de Guadalajara.

2013. "Agenda de competitividad de Puerto Vallarta". Disponible en:

http://secturjal.jalisco.gob.mx/sites/secturjal.jalisco.gob.mx/files/u16/agenda_puerto vallarta.pdf

Scartascini, Gabriela, y Nuñez, Patricia.

2008. "De pueblo en pueblo: alternativas para un destino de sol y playa". En Orozco, Javier; Nuñez, Patricia y Virgen, Carlos. Desarrollo turístico y sustentabilidad social. México. Ed. Miguel A. Purrua: 171-188

Sforzi, Fabio.

2001. "La teoría marshalliana para explicar el desarrollo local". En Rodríguez, Fermín. Manual de desarrollo local. España. Ed. TREA: 13-32.

TPDS. (Tecnología y Planeación para el Desarrollo Sostenible)

2012. "Plan de Manejo para la presa Cajon de Peñas". Recuperado el 26 de 06 de 2017, Disponible en www.cofemersimir.gob.mx/expediente/5730/mir/14667/anexo/560413

Tello, Carlos.

2013. "Desarrollo versus conservación en la disputa por los humedales del bosque tropical seco: el caso de la reserva de la Biosfera Chamela- Cuixmala, Jalisco, México". Revista Interciencia, 38 (3).

Tello, Carlos.

2014a. "La colonización de la costa de Jalisco". Revista Relaciones (140).

Tello, Carlos.

2014b. "Los señores de la costa: historias de poder en Careyes y Cuixmala". México. Ed. Grijalbo.

Tinbergen, Jaen

1989. La planeación del desarrollo. México. Ed. F. C. E.

UNAM (Universidad Nacional Autonoma de México) 
2018. La reserva de la Biosfera Chamela-Cuixmala. Disponible en http://www.ibiologia.unam.mx/ ebchamela/www/reserva.html

Vazquez Barquero, Antonio.

2001. "La política de desarrollo local en Europa". En Rodríguez, Fermín. Manual de Desarrollo Local. España: Ediciones TREA: 42- 60.

Vera, Fernando.

1994. "El modelo turístico mediterráneo español: agotamiento y estrategia de reestructuración". Revista Papers de turisme (14-15): 131-147.

Virgen, Carlos.

2014. El turismo en Puerto Vallarta: pasado y presente. México. Ed. UFPR- CONAET.

Yáñez, Agustín

1996. La tierra prodiga. México, Ed. F. C. E.

\section{Notas}

1 La historia de Acapulco esta definida por la Nao de China que fue el vehículo comercial con Asia del imperio español y la colonia de América, que lo convirtió en el principal puerto marítimo del pacifico mexicano. Sera revitalizado a partir del turismo en la década de 1940 convirtiéndolo en un icono mundial.

2 Yáñez es reconocido como uno de los más importantes escritores mexicanos del siglo XX, además de haber sido político que culmino su carrera como Gobernador de Jalisco. Tener la oportunidad de conocer de primera mano la situación de la costa de Jalisco a manera de novela, es muy gratificante y aleccionador. 